\title{
Forage Preferences of Livestock in the Arid Lands of Northern Kenya
}

\author{
W.J. LUSIGI, E.R. NKURUNZIZA, AND S. MASHETI
}

\section{Abstract}

The desirability of forage plants by livestock or wildlife is an important consideration in evaluating suitability of the range for grazing. This desirability may also in some cases be used in determining range condition. In many range types periodic determination of plant species composition provides the best indication of long-term trends. Evaluation of the effects of grazing on range flora usually requires that the vegetation be assigned to significant groups. This work represents the first attempt to make this kind of classification for the arid zone of northern Kenya in the study area of the UNESCO Integrated Project in Arid Lands (IPAL). It was required for the preparation of grazing plans for the largely nomadic pastoralists there. Preferences for 250 plant species have been assessed for camels, sheep, goats, and cattle. They are based on the best information presently available, and forms our basis for the classification of range condition for 147 range types in the study area.

The arid lands of Kenya, which cover over $75 \%$ of that country, are being seriously degraded as a result of increased pressures from human livestock populations, periodic droughts, and an imbalance between modern and traditional pastoral practices. The productivity of the range has been so reduced that pastoralists are under constant threat of famine. The Integrated Project on Arid Lands (IPAL), of which this work is part, was established by UNESCO to reverse this degradation.

Research on the plant species preferred by livestock in Kenya, particularly in the arid north, has been limited. Since the establishment of the IPAL program in 1976, the published vegetational work has consisted of an annotated vegetation map (Herlocker 1979a), problems concerning the status of montane and other forests (Synott 1979a,b; Herlocker 1979b), an annotated check-list of the plants of Mount Kulal, Kenya (Hepper et al. 1981), feeding observations of camels, cattle, sheep, and goats (Field 1978, 1979; Kayongo-Male and Field 1981; Lewis 1977) and herb-layer productivity in relation to rainfall (Herlocker and Dolan 1980a, 1980b, Walther and Herlocker 1980). Work on woodland availability, productivity and exploitation has also been reported (Lusigi 1981).

The importance of forage plants for both livestock and wildlife is determined by their availability, palatability, and nutritive value. A combination of various levels of these 3 factors should be considered in the condition rating of a particular range site. In more productive rangelands and pastures in Kenya, work has proceeded from determining the availability of forage species to analysis of the nutritive value of preferred forage species (Dougall) and Bogdan 195la,b; Dougall et al. 1964; Field 1975; Gwynne 1969; Taerum 1970). In the less productive rangelands in the arid zone, information available on the nutritive value of indigenous forage species and their preference for livestock is still very scanty except for a generalized review of browse (Lamprey et al. 1980). The objective of this study was to classify the preference of livestock for

Authors are project co-ordinator and range ecologist, plant ecologist and plant taxonomist with UNESCO Integrated Project on Arid Lands, UNESCO Regional Office for Science and Technology for Africa, P.O. Box 30592, Nairobi, Kenya.

The authors wish to acknowledge contributions made to this study by all IPAL Scientists and Field Support Staff.

Manuscript accepted December 15, 1983 all major forage plants in the area so that range condition and trend can be assessed.

\section{Study Area}

The IPAL study area has been described in the many technical reports published by the project (Lusigi 1981, 1983). It covers an area of $23,000 \mathrm{~km}^{2}$ in the Marsabit District of Northern Kenya, situated between 5 major mountain ranges: Hurri Hills, Marsabit Mountain, Ndoto mountain range, Mount Kulal, and four important water catchment systems in this arid environment (Herlocker 1979).

The vegetation cover of the area is varied. There are small montane forests at the peak of the mountains, mist forests on hills, and perennial grasslands on lower slopes. In the lowlands are ground water forests, semideciduous woodlands, deciduous woodlands, semiarid to arid bushlands and shrublands, arid dwarf shrublands, annual grasslands, and barren lands. With exception of the mountains which receive about $700 \mathrm{~mm}$ average annual rainfall, the rest of the area receives about $200 \mathrm{~mm}$. The rainfall is highly erratic.

The area is inhabited by nomadic and seminomadic tribes, which include the Rendille, Samburu, Gabbra, Borana, and Turkana. Apart from minimal agricultural activities on Marsabit mountain, the entire nomadic population derives its livelihood from livestock. The major livestock are camels, sheep, goats, and cattle.

The main species of wild grazing herbivores in the study area are Beisa oryx (Oryx beisa beisa) and Grevy's zebra (Equus grevyi). Other wild herbivores include dik dik (Rhynchotragus guentheri), genoruk (Litocranius walleri), Grant's gazelle (Gazella granti), reticulated giraffe (Giraffa camelopardalis reliculata), rhinoceros (Diceros bicornis), and elephant (Loxodonta africana). Other animals include lion (Panthera leo), leopard (Panthera Pardus), and chcctah (Acinonyxjubatus).

Numerically, sheep and goats are most abundant, followed by cattle and camels. The latest aerial survey of the study area estimated 287,040 sheep and goats, 56,810 cattle, 41,400 camels, 1,150 donkeys, 1,840 oryxes, 1,380 zebras, 1,610 giraffes, 3,600 Grant's gazelles, 1,150 gerenuks, and 2,070 ostriches, a total of just under 400,000 animals (Lusigi 1981). The livestock in the study area (excluding donkeys) comprises about $44 \%$ of the total livestock estimated for the entire Marsabit District.

\section{Materials and Methods}

Preferences of wild ungulates for various plants have been discussed by Talbot (1962), Stewart (1966), and Field (1968). They consist mainly of direct feeding observations of plant species actually ingested by the particular animal species, examination of diet samples collected through oesophogeal and rumen fistulas, analyses of stomach contents by cropping animals, and microhistological analyses of fecal material.

The method most commonly used by IPAL livestock scientists has been the direct observation of plants actually eaten. This method is improved by determining the period of time an animal spends feeding on a particular plant species (Field 1978). Data have been collected in various parts of the study area both in the dry and rainy season. The desirability of a plant species is best determined 
during the rainy season, when plants are at optimal growth stages. The desirability of a species in the dry season largely reflects its availability (Field 1968). Data obtained from the above observations are supplemented by interviews with herdsmen who have fairly accurate knowledge of plants eaten by their livestock. Analyses done on fecal material of cattle (Kaycngo-Male and and Field 1981) and goats (Said 1980) were incorporated into this study.

This paper is a synthesis of available data in published and unpublished articles, and interviews with local people in the IPAL study area.

Food preferences have been grouped into 4 categories-Very Desirable (VD), Desirable (D), Intermediate (I), and Undesirable (U). Very Desirable plants were those observed and reported to be eaten every time an animal encountered them. Desirable plants were those constituting $80 \%$ of the animal's diet (Kayongo-Male and Field 1983) and, together with the very desirables, accounted for $80 \%$ of the animal's feeding time. Intermediate species were those comprising the remaining $20 \%$ of the animal's diet and feeding time, but this category also includes those plants known to be eaten only when there was little or no alternative forage. Undesirables were those with no observed utilization. The last category includes toxic and noxious plants. However, a plant species preferred by an animal at a given time may, in some circumstances, prove to be toxic, especially when eaten in large quantities. Others may be toxic at particular growth stages. Caution must therefore be exercised in interpreting these data, since no attempt is made to give any information on toxicity or nutritive value in terms of chemical composition.

A total of 256 plant species consisting of 80 trees and large shrubs, 34 dwarf shrubs, 22 perennial grasses, 68 herbs, 38 annual grasses and sedges and 14 creepers and climbers were examined.

\section{Results and Discussion}

\section{Seasonal Use of Plants}

Since rainfall in the IPAL study area is low and temperatures high (Edwards et al. 1979), a large number of annuals and deciduous plant species are present. A fair number of perennial species have also been observed on the study area which are utilized in both the dry and rainy seasons. During the drier parts of the year, annuals may still form an important part of the diet of livestock, in standing dead form or litter. The desirability of a plant species, or its undesirability, may reflect its availability rather than palatability. For example, the desirability of most Cadeba species seems to improve in the dry season for camels; and though Balanites aegyptiace is recorded as undesirable in the wet season for the same livestock species, it is intermediate in the dry season (Table 1), because it remains green long after the desirable species have lost their leaves.

Table 1. Food preference of various livestock species in the Integrated Project on Arid Lands (1.P.A.L.)Study Area in the wet and dry seasons. (VD - Very desirable; D - Desirable; I - Intermediate; U - Undesirable)

\begin{tabular}{|c|c|c|c|c|c|c|c|c|}
\hline \multirow[b]{2}{*}{ Plant Species } & \multicolumn{2}{|c|}{ Camels } & \multicolumn{2}{|c|}{ Sheep } & \multicolumn{2}{|c|}{ Goats } & \multicolumn{2}{|c|}{ Cattle } \\
\hline & Wet & Dry & Wet & Dry & Wet & Dry & Wet & Dry \\
\hline \multicolumn{9}{|l|}{ Trees and Large Shrubs } \\
\hline Acacia brevispica & $\mathbf{D}$ & $D$ & $\mathbf{U}$ & I & $\mathbf{D}$ & $D$ & $U$ & $U$ \\
\hline Acacia eletior & D & $\mathrm{D}$ & $\mathbf{U}$ & $\mathrm{U}$ & $\mathrm{U}$ & 1 & $\mathbf{U}$ & $\mathrm{U}$ \\
\hline Acacia horrida & $\mathbf{U}$ & $\mathbf{U}$ & $\mathbf{U}$ & $\mathrm{U}$ & $\mathrm{U}$ & $\mathrm{U}$ & $\mathrm{U}$ & $\mathrm{U}$ \\
\hline Acacia mellifera & I & $\mathbf{U}$ & $\mathrm{U}$ & D & D & D & $\mathrm{U}$ & $\mathbf{U}$ \\
\hline Acacia nubica & $\mathbf{U}$ & I & $\mathrm{U}$ & $\mathrm{U}$ & $\mathrm{U}$ & I & $\mathbf{U}$ & $\mathrm{U}$ \\
\hline Acacia paolii & 1 & $\mathbf{U}$ & $\mathbf{U}$ & $\mathrm{U}$ & I & $\mathrm{U}$ & $\mathbf{U}$ & $\mathrm{U}$ \\
\hline Acacia reficiens & 1 & $\mathbf{U}$ & U & $\mathrm{U}$ & I & I & $\mathbf{U}$ & $\mathrm{U}$ \\
\hline Acacia senegal & $\mathbf{U}$ & $\mathbf{U}$ & $\mathrm{U}$ & $\mathrm{U}$ & D & $\mathrm{U}$ & $\mathrm{U}$ & $\mathrm{U}$ \\
\hline Acacia seyal var seyal & $\mathbf{U}$ & I & $\mathbf{U}$ & $\mathrm{U}$ & $\mathrm{U}$ & 1 & $\mathbf{U}$ & $\mathbf{U}$ \\
\hline Acacia tortilis & D & VD & $\mathrm{U}$ & D & I & VD & $\mathrm{U}$ & $\mathrm{U}$ \\
\hline Balanites aegyptiaca & $\mathrm{U}$ & I & $\mathrm{U}$ & $\mathbf{U}$ & $\mathrm{U}$ & $\mathrm{U}$ & $\mathrm{U}$ & $\mathbf{U}$ \\
\hline Boscia angustifolia & $\mathrm{U}$ & $\mathbf{U}$ & $\mathbf{U}$ & $\mathrm{U}$ & $\mathrm{U}$ & $\mathrm{U}$ & $\mathrm{U}$ & I \\
\hline Boscia coriacea & $\mathrm{U}$ & I & $\mathbf{U}$ & $\mathrm{U}$ & $\mathrm{U}$ & $\mathrm{U}$ & $\mathrm{U}$ & $\mathrm{U}$ \\
\hline Bascia minimifolia & $\mathbf{U}$ & $\mathbf{U}$ & $\mathbf{U}$ & $\mathbf{U}$ & $\mathbf{U}$ & $\mathrm{U}$ & $\mathrm{U}$ & $\mathrm{U}$ \\
\hline Boswellia hildebrandtii & VD & D & $\mathbf{U}$ & I & VD & D & $\mathrm{U}$ & $\mathrm{U}$ \\
\hline Cadaba farinosa & $\mathrm{U}$ & $\mathrm{D}$ & $\mathrm{U}$ & $\mathbf{U}$ & $\mathbf{U}$ & D & $\mathrm{U}$ & $\mathrm{U}$ \\
\hline Cadaba glandulosa & I & $\mathrm{D}$ & $\mathrm{U}$ & D & $\mathrm{U}$ & D & $\mathrm{U}$ & $\mathrm{U}$ \\
\hline Cadaba mirabilis & $\dot{U}$ & $D$ & U & $\mathrm{U}$ & $\mathbf{U}$ & 1 & $U$ & $U$ \\
\hline Cadaba ruspolii & $\mathrm{U}$ & I & $\mathrm{U}$ & $\mathbf{U}$ & $\mathbf{U}$ & $\mathbf{U}$ & $\mathrm{U}$ & $\mathrm{U}$ \\
\hline Cadaba sp.* & $\mathbf{U}$ & i & $\mathrm{U}$ & $\mathbf{U}$ & $\mathrm{D}$ & I & $\mathbf{U}$ & $\mathrm{U}$ \\
\hline Cambretun aculeatum & D & $\mathrm{D}$ & $\mathrm{U}$ & I & D & D & $\mathrm{U}$ & $\mathrm{U}$ \\
\hline Commiphora africana & $\mathrm{D}$ & $\mathrm{U}$ & $\mathrm{U}$ & $\mathrm{U}$ & $\mathbf{D}$ & 1 & $\mathrm{U}$ & $\mathrm{U}$ \\
\hline Commiphora boiviniana & l & $\mathrm{U}$ & $\mathrm{U}$ & $\mathbf{U}$ & I & $\mathrm{U}$ & $\mathrm{U}$ & $\mathrm{U}$ \\
\hline Commiphora candidula & $\mathbf{D}$ & $\mathbf{U}$ & U & $\mathbf{U}$ & D & $\mathrm{U}$ & $\mathbf{U}$ & U \\
\hline Commiphora flaviflora & $\mathrm{D}$ & $\mathbf{U}$ & $\mathrm{U}$ & $\mathbf{U}$ & D & $\mathbf{U}$ & $\mathbf{U}$ & $\mathrm{U}$ \\
\hline Commiphora paolii & I & $\mathbf{U}$ & $\mathrm{U}$ & $\mathbf{U}$ & I & $\mathrm{U}$ & $\mathbf{U}$ & $\mathrm{U}$ \\
\hline \multicolumn{9}{|l|}{ Commiphora $\mathrm{P} / \mathrm{Y}^{*}$ (Walther et al. } \\
\hline 1980) & 1 & $\mathbf{U}$ & $\mathrm{u}$ & I & D & I & $\mathbf{U}$ & $\mathrm{U}$ \\
\hline Commiphora rostrata & I & $\mathrm{U}$ & $\mathrm{U}$ & $\mathbf{U}$ & 1 & $\mathrm{U}$ & $\mathrm{U}$ & $\mathrm{U}$ \\
\hline Commiphora sp. (green bark)* & D & I & $\mathrm{U}$ & $\mathrm{U}$ & D & I & $\mathrm{U}$ & $\mathrm{U}$ \\
\hline \multicolumn{9}{|l|}{ Commiphora sp. (red \& white } \\
\hline bark)* & I & $\mathbf{U}$ & $\mathrm{U}$ & $\mathbf{U}$ & I & I & $\mathbf{U}$ & $\mathrm{U}$ \\
\hline Cordia crenata & $\mathbf{U}$ & I & $\mathrm{U}$ & $\mathbf{U}$ & $\mathbf{U}$ & I & $\mathrm{U}$ & $\mathrm{U}$ \\
\hline Cordia sinensis & $\mathrm{D}$ & $\mathbf{U}$ & $\mathrm{U}$ & $\mathbf{U}$ & $\mathrm{D}$ & $\mathrm{U}$ & $\mathbf{U}$ & U \\
\hline Croton dichogamus & $\bar{U}$ & $\mathbf{U}$ & $\mathrm{U}$ & $\mathbf{U}$ & $\mathbf{U}$ & $\mathrm{U}$ & $U$ & $\mathrm{U}$ \\
\hline Dobera glabra & $\mathbf{U}$ & $\mathbf{U}$ & $\mathrm{U}$ & $\mathbf{U}$ & $\mathbf{U}$ & $\mathbf{U}$ & $\mathrm{U}$ & $\mathrm{U}$ \\
\hline Euphorbia cuneata & I & $\mathrm{U}$ & $\mathrm{U}$ & $\mathbf{U}$ & D & $\mathbf{U}$ & $\mathrm{U}$ & $\mathrm{U}$ \\
\hline Grewia bicolor & I & 1 & $\mathrm{U}$ & I & I & I & $\mathbf{U}$ & I \\
\hline Grewia trembensis & D & D & U & $\mathbf{U}$ & D & D & I & $\mathrm{U}$ \\
\hline Grewia tenax & I & $\mathbf{U}$ & $\mathbf{U}$ & $\mathbf{U}$ & D & D & $\mathrm{U}$ & $\mathrm{U}$ \\
\hline Grewia trichocarpa & I & $\mathrm{D}$ & $\mathrm{U}$ & $\mathbf{U}$ & $\mathbf{U}$ & D & $\mathrm{U}$ & $\mathrm{U}$ \\
\hline
\end{tabular}


Table 1. Continued.

\begin{tabular}{|c|c|c|c|c|c|c|c|c|}
\hline \multirow[b]{2}{*}{ Plant Spccics } & \multicolumn{2}{|c|}{ Camels } & \multicolumn{2}{|c|}{ Sheep } & \multicolumn{2}{|c|}{ Goats } & \multicolumn{2}{|c|}{ Cattle } \\
\hline & Wet & Dry & Wet & Dry & Wet & Dry & Wet & Dry \\
\hline Grewia villosa & D & $\mathrm{U}$ & $\mathrm{U}$ & $\mathbf{U}$ & $\mathrm{D}$ & $\mathbf{U}$ & $\mathbf{U}$ & $\mathrm{U}$ \\
\hline Jatropha dichtar & $\mathrm{U}$ & $\mathrm{U}$ & $\mathbf{U}$ & $\mathbf{U}$ & $\mathbf{U}$ & $\mathrm{U}$ & $\mathrm{U}$ & $\mathrm{U}$ \\
\hline Lawsonia inermis & $\mathrm{D}$ & D & $\mathbf{U}$ & I & $\mathrm{D}$ & $\mathrm{D}$ & $\mathrm{U}$ & $\mathrm{U}$ \\
\hline Lycium europeaum & I & $\mathrm{U}$ & $\mathrm{U}$ & $\mathbf{U}$ & $\mathbf{u}$ & $\mathrm{U}$ & $\mathrm{U}$ & $\mathrm{U}$ \\
\hline Maerua crassifolia & $\mathrm{U}$ & $\mathrm{D}$ & $\mathrm{U}$ & $\mathbf{U}$ & $\mathrm{U}$ & D & $\mathrm{U}$ & $\mathrm{U}$ \\
\hline Maerua oblongifolia & $\mathrm{D}$ & D & $\mathrm{U}$ & $\mathbf{U}$ & $\mathbf{U}$ & I & $\mathrm{U}$ & $\mathrm{U}$ \\
\hline Maytenus senegalensis & $\mathrm{U}$ & $\mathrm{U}$ & $\mathbf{U}$ & $\mathrm{U}$ & D & D & $\mathrm{U}$ & $\mathrm{U}$ \\
\hline Maringa sp.* & $\mathrm{U}$ & $\mathbf{U}$ & $\mathrm{U}$ & $\mathrm{U}$ & $\mathrm{U}$ & $\mathrm{U}$ & $\mathrm{U}$ & $\mathrm{U}$ \\
\hline Opilia campenstris & 1 & $\mathbf{U}$ & $\mathbf{U}$ & $\mathrm{U}$ & $\mathrm{D}$ & I & $\mathrm{U}$ & $\mathrm{U}$ \\
\hline Ormocarpum trichocarpum & $\mathbf{U}$ & $\mathrm{U}$ & $\mathrm{U}$ & $\mathrm{U}$ & $\mathrm{U}$ & $\mathrm{U}$ & $\mathrm{U}$ & $\mathrm{U}$ \\
\hline Premna resinosa & $\mathrm{D}$ & $\mathrm{D}$ & $\mathrm{U}$ & I & VD & $\mathrm{D}$ & $\mathrm{U}$ & $\mathrm{U}$ \\
\hline Rennea triphylla & $\mathbf{U}$ & $\mathrm{U}$ & $\mathrm{U}$ & $\mathrm{U}$ & $\mathrm{U}$ & $\mathrm{U}$ & $\mathrm{U}$ & U \\
\hline Salvadora persica & $\mathbf{I}$ & $\mathrm{D}$ & $\mathbf{U}$ & $\mathrm{U}$ & $\mathrm{U}$ & I & $\mathrm{U}$ & $\mathrm{U}$ \\
\hline Securinega virosa & $\mathbf{U}$ & $\mathbf{U}$ & $\mathrm{U}$ & $\mathrm{U}$ & $\mathrm{U}$ & $\mathrm{U}$ & $\mathrm{U}$ & $\mathrm{U}$ \\
\hline Sesamothamnus rivae & $\mathrm{U}$ & $\mathbf{U}$ & $\mathrm{U}$ & $\mathrm{U}$ & $\mathrm{D}$ & $\mathrm{U}$ & $\mathrm{U}$ & $\mathrm{U}$ \\
\hline Solanum arundo & $\mathrm{U}$ & $\mathrm{U}$ & $\mathrm{U}$ & $\mathrm{U}$ & $\mathrm{U}$ & $\mathrm{U}$ & $\mathrm{U}$ & $\mathrm{U}$ \\
\hline Sterculia stenocarpa & $\mathrm{U}$ & $\mathrm{U}$ & $\mathrm{U}$ & $\mathrm{U}$ & $\mathrm{U}$ & $\mathrm{U}$ & $\mathrm{U}$ & $\mathrm{U}$ \\
\hline Terminalis mollis & $\mathrm{D}$ & $\mathrm{U}$ & $\mathrm{U}$ & $\mathrm{U}$ & VD & $\mathrm{U}$ & $\mathrm{U}$ & $\mathrm{U}$ \\
\hline Terminalis orbicularis & I & $\mathrm{U}$ & $\mathrm{U}$ & $\mathrm{U}$ & $\mathrm{D}$ & $\mathrm{U}$ & $\mathrm{U}$ & $\mathrm{U}$ \\
\hline Trema orientalis & I & $\mathrm{U}$ & $\mathbf{U}$ & $\mathrm{U}$ & I & $\mathrm{U}$ & $\mathrm{U}$ & $\mathrm{U}$ \\
\hline Vangueria linearisepala & $\dot{\mathrm{U}}$ & $\mathrm{U}$ & $\mathrm{U}$ & $\mathrm{U}$ & $\mathrm{U}$ & $\mathrm{U}$ & $\mathrm{U}$ & $\mathrm{U}$ \\
\hline Wrigtia demartianiana & $\mathrm{U}$ & $\mathrm{U}$ & $\mathrm{U}$ & $\mathrm{U}$ & $\mathrm{U}$ & $\mathrm{U}$ & $\mathrm{U}$ & $\mathrm{U}$ \\
\hline Ziziphus mautiana & $n$ & $\mathrm{D}$ & $\mathrm{U}$ & $\mathrm{U}$ & U & I & $\mathrm{U}$ & $\mathrm{U}$ \\
\hline
\end{tabular}

Shrubs

Asparagus aethiopicus

Asparagus buchananii

Asparagus setaceus

Aspilia mossambicensis

Blepharispermum fruticosum

Clerodendrum myricoides

Cyclocheilon erianthemum

Euclea schimperi

Euphorbia scheffleri

Hibiscus aponeurus

Hibiscus sp.*

Jatropha parvifolia

Jatropha villosa

Lippia sp.

Osyris abyssinica

Tinnea aethiopica

Tinnea sp.*

Veronia brachycalx

$\begin{array}{cc}\text { U } & \text { U } \\ \text { U } & \mathrm{U} \\ \mathrm{U} & \mathrm{U} \\ \mathrm{D} & \mathrm{U} \\ \mathrm{U} & \mathrm{U} \\ \mathrm{U} & \mathrm{U} \\ \mathrm{I} & \mathrm{U} \\ \mathrm{U} & \mathrm{U} \\ \mathrm{U} & \mathrm{U} \\ \mathrm{U} & \mathrm{I} \\ \mathrm{I} & \mathrm{I} \\ \mathrm{U} & \mathrm{U} \\ \mathrm{U} & \mathrm{U} \\ \mathrm{U} & \mathrm{U} \\ \mathrm{U} & \mathrm{U} \\ \mathrm{U} & \mathrm{U} \\ \mathrm{U} & \mathrm{U} \\ \mathrm{I} & \mathrm{U}\end{array}$

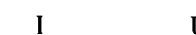

\begin{tabular}{|c|c|c|}
\hline $\mathrm{U}$ & $\mathbf{U}$ & $\mathbf{U}$ \\
\hline I & $\mathrm{U}$ & $\mathrm{U}$ \\
\hline $\mathrm{U}$ & $\mathbf{U}$ & $\mathbf{U}$ \\
\hline $\mathrm{U}$ & I & $\mathrm{U}$ \\
\hline I & $\mathbf{U}$ & $\mathrm{U}$ \\
\hline I & $\mathbf{U}$ & $\mathrm{U}$ \\
\hline $\mathrm{U}$ & $\mathrm{U}$ & $\mathrm{U}$ \\
\hline $\mathrm{U}$ & $\mathrm{U}$ & $\mathbf{U}$ \\
\hline $\mathbf{U}$ & $\mathrm{U}$ & $\mathbf{U}$ \\
\hline D & $\mathbf{U}$ & 1 \\
\hline 1 & $\mathbf{U}$ & I \\
\hline $\mathbf{U}$ & $U$ & $\mathbf{U}$ \\
\hline $\mathbf{U}$ & $\mathbf{U}$ & $\mathbf{U}$ \\
\hline I & $\mathrm{U}$ & $\mathrm{D}$ \\
\hline $\mathrm{U}$ & $\mathrm{U}$ & $\mathrm{U}$ \\
\hline I & $\mathrm{U}$ & $\mathrm{U}$ \\
\hline D & $\mathrm{U}$ & $\mathrm{U}$ \\
\hline $\mathbf{U}$ & $U$ & $\mathrm{U}$ \\
\hline
\end{tabular}

Dwarf shrubs

Acalypha fruticosa

Aloe sp.*

Barleria acanthoides

Barleria eranthemoides

Barleria proxima

Barleris sp.*

Crotalaria fasicularis

Crotalaris laburnifolia

Daysphaera prosirata

Duosperma cremophilum

Euphorbia heterochroma

Helinus integrifolia

Heliotropium albohispidum

Indigofera cliffordiana

Indofera spinosa

Ipomoea kituensis

Justicia caerulca

Justicia fischeri

Justicia odora

Justicia pinguor

Kleinia kleinioidea

Lantana rhodesiensis

Leucas tomentosa

Ocimum americanum

Ocimum sp*

Ocimum suave

Plectranthus igniarus

$\begin{array}{rrr}\text { I } & \text { U } & \\ \text { U } & \text { I } & \\ \text { I } & \text { U } & \\ D & U & \\ U & I & \\ U & U & \\ D & U & \\ U & U & \\ U & U & \\ D & D & \\ D & I & \\ U & U & \\ U & U & \\ I & D & U \\ U & U & U \\ D & I & U \\ \text { VD } & \text { D } & \text { U } \\ D & \text { U } & \end{array}$

\begin{tabular}{|c|c|c|c|c|c|c|c|}
\hline D & D & D & I & D & I & $\mathrm{U}$ & D \\
\hline $\mathrm{U}$ & $\mathrm{U}$ & $\mathbf{U}$ & $\mathrm{U}$ & $\mathrm{U}$ & $\mathbf{U}$ & $\mathrm{U}$ & $\mathrm{U}$ \\
\hline D & 1 & $\mathbf{U}$ & $\mathrm{U}$ & D & I & I & I \\
\hline D & D & $\mathrm{U}$ & I & D & $\mathrm{D}$ & $\mathrm{U}$ & I \\
\hline D & D & D & $\mathrm{D}$ & D & D & $\mathrm{U}$ & $\mathrm{U}$ \\
\hline D & $\mathbf{U}$ & D & $\mathbf{U}$ & D & $\mathrm{U}$ & $\mathrm{U}$ & $\mathrm{U}$ \\
\hline $\mathrm{U}$ & $\mathbf{U}$ & $\mathbf{U}$ & $\mathrm{U}$ & I & $\mathrm{U}$ & $\mathrm{U}$ & $\mathrm{U}$ \\
\hline 1 & D & I & D & I & $\mathrm{D}$ & I & D \\
\hline D & D & $\mathbf{U}$ & $\mathrm{U}$ & $\mathrm{U}$ & 1 & $\mathrm{U}$ & $\mathrm{U}$ \\
\hline I & I & $\mathbf{U}$ & $\mathrm{U}$ & I & I & I & $\mathrm{U}$ \\
\hline $\mathbf{U}$ & $\mathbf{U}$ & $\mathrm{U}$ & $\mathrm{U}$ & $\mathbf{U}$ & $\mathbf{U}$ & $\mathrm{U}$ & $\mathbf{U}$ \\
\hline D & $D$ & $\mathrm{U}$ & $\mathrm{U}$ & D & $\mathrm{D}$ & $\mathrm{U}$ & $\mathrm{U}$ \\
\hline D & I & D & I & D & I & $\mathrm{U}$ & $\mathrm{U}$ \\
\hline D & U & $\mathrm{D}$ & $\mathbf{U}$ & D & $\mathrm{U}$ & $\mathrm{U}$ & $\mathrm{U}$ \\
\hline VD & VD & $\mathrm{D}$ & I & VD & I & l & $\mathrm{U}$ \\
\hline $\mathrm{U}$ & $\mathrm{U}$ & $\mathbf{U}$ & $\mathrm{U}$ & $\mathrm{U}$ & $\mathrm{U}$ & $\mathbf{U}$ & $\mathbf{U}$ \\
\hline VD & D & I & I & VD & D & $\mathbf{U}$ & 1 \\
\hline D & I & I & $\mathrm{U}$ & D & 1 & $\mathrm{U}$ & $\mathrm{U}$ \\
\hline $\mathrm{D}$ & I & I & $\mathrm{U}$ & $\mathrm{U}$ & I & $\mathrm{U}$ & $\mathrm{U}$ \\
\hline $\mathrm{D}$ & D & D & D & D & D & $\mathrm{U}$ & I \\
\hline $\mathrm{U}$ & $\mathrm{U}$ & $\mathrm{U}$ & $\mathrm{U}$ & $\mathrm{U}$ & $\mathrm{U}$ & $\mathrm{U}$ & $\mathrm{U}$ \\
\hline $\mathrm{U}$ & I & $\mathrm{U}$ & I & $\mathrm{U}$ & 1 & $\mathrm{U}$ & I \\
\hline $\mathrm{U}$ & $\mathrm{U}$ & $U$ & $\mathrm{U}$ & $\mathrm{U}$ & $\mathrm{U}$ & $\mathrm{U}$ & $\mathbf{U}$ \\
\hline $\mathrm{U}$ & I & $U$ & I & $\mathrm{U}$ & I & $\mathrm{U}$ & 1 \\
\hline $\mathrm{U}$ & I & $\mathrm{U}$ & I & $\mathrm{U}$ & I & $\mathrm{U}$ & I \\
\hline $\mathrm{U}$ & 1 & $\mathrm{U}$ & I & $\mathrm{U}$ & I & $\mathrm{U}$ & I \\
\hline $\mathrm{D}$ & D & $\mathrm{U}$ & I & D & D & $\mathrm{D}$ & I \\
\hline
\end{tabular}


Table 1. Continued.

\begin{tabular}{|c|c|c|c|c|c|c|c|c|}
\hline \multirow[b]{2}{*}{ Plant Species } & \multicolumn{2}{|c|}{ Camels } & \multicolumn{2}{|c|}{ Sheep } & \multicolumn{2}{|c|}{ Goats } & \multicolumn{2}{|c|}{ Cattle } \\
\hline & Wet & Dry & Wet & Dry & Wet & Dry & Wet & Dry \\
\hline Salsola dendroides & $\mathbf{U}$ & D & $\mathrm{u}$ & $\mathrm{U}$ & $\mathrm{U}$ & I & $\mathbf{u}$ & $\mathbf{U}$ \\
\hline Satureja abyssinica & I & $\mathrm{D}$ & I & D & I & D & u & D \\
\hline Seddera hirsuta & $\mathrm{U}$ & 1 & $\mathbf{U}$ & $\mathrm{U}$ & $\mathrm{U}$ & I & $\mathrm{u}$ & $\mathrm{U}$ \\
\hline Sericocomopsis hilderbrandtii & D & $\mathrm{D}$ & D & I & I & D & $\mathrm{u}$ & D \\
\hline Sericocomopsis pallida & I & D & $\mathbf{U}$ & I & I & D & $\mathrm{u}$ & 1 \\
\hline Solanum sp. (IPAL)* & I & $\bar{U}$ & $\mathrm{U}$ & $\mathrm{U}$ & $\mathbf{U}$ & $\mathbf{U}$ & $\mathbf{u}$ & $\mathbf{U}$ \\
\hline Suaeda monoica & $\mathrm{U}$ & D & $\mathbf{U}$ & $\mathrm{U}$ & $\mathbf{U}$ & $\mathbf{U}$ & $\mathbf{u}$ & $\mathbf{U}$ \\
\hline \multicolumn{9}{|l|}{ Perennial grasses } \\
\hline Botheiochloa inseculpta & I & $\mathrm{u}$ & VD & D & D & $\mathbf{u}$ & VD & $\mathrm{D}$ \\
\hline Brachiaria sp. (Olturot)* & $\mathbf{U}$ & 1 & D & D & I & I & D & D \\
\hline Brachiaria sp.* & U & $\mathrm{U}$ & D & D & I & $\mathbf{U}$ & $\mathrm{D}$ & $\mathrm{D}$ \\
\hline Cenchrus pennisetiformis & I & $\mathrm{U}$ & D & $\mathrm{U}$ & $\mathrm{U}$ & $\mathbf{u}$ & $\mathrm{D}$ & $\mathrm{U}$ \\
\hline Chrysopogon plumulosus & $\dot{U}$ & $\mathrm{U}$ & D & D & $\mathrm{U}$ & $\mathbf{U}$ & $\mathrm{D}$ & D \\
\hline Cynodon dactylon & $\mathrm{U}$ & $\mathrm{U}$ & $\mathrm{D}$ & D & $\mathrm{D}$ & I & $\mathrm{D}$ & $\mathrm{D}$ \\
\hline Dactyloctenium aegyptium & $\mathrm{U}$ & $\mathrm{U}$ & D & $\mathrm{U}$ & 1 & $\dot{U}$ & $\mathrm{D}$ & U \\
\hline Digitaria abyssinica & $\mathrm{U}$ & $\mathrm{U}$ & D & D & D & D & $\mathrm{D}$ & D \\
\hline Digitaria scalarum & $\mathrm{U}$ & $\mathrm{U}$ & D & D & D & D & D & $\mathrm{D}$ \\
\hline Hyparrhenia hirta & $\mathbf{U}$ & $\mathrm{U}$ & D & I & $\mathrm{U}$ & $\mathrm{U}$ & $\mathrm{D}$ & I \\
\hline Leptochloa abtusifolia & $\mathbf{U}$ & $\mathrm{U}$ & D & D & D & I & D & D \\
\hline Sehima nervosum & $\mathrm{U}$ & $\mathrm{U}$ & D & D & $\mathrm{D}$ & $\mathrm{D}$ & $\mathrm{D}$ & $\mathrm{D}$ \\
\hline Setaria sp.* & $\mathbf{U}$ & $\mathbf{U}$ & D & D & D & I & D & D \\
\hline Sporolobus agrostoides & $\mathrm{U}$ & I & $\mathrm{D}$ & D & I & 1 & D & D \\
\hline Sporobolus fimbriatus & $\mathrm{U}$ & $\dot{U}$ & $\mathrm{D}$ & D & i & $\mathrm{D}$ & D & $\mathrm{D}$ \\
\hline Sporobolus hervolus & $\mathrm{U}$ & I & $\mathrm{D}$ & I & $\mathrm{U}$ & I & $\mathrm{D}$ & $\mathrm{D}$ \\
\hline Sporobolus nervosus & $\mathrm{U}$ & $\mathrm{U}$ & D & D & $\mathrm{U}$ & $\mathrm{U}$ & D & $\mathrm{D}$ \\
\hline Sporobolus sp. (Maikona)* & $\mathrm{U}$ & U & D & D & $\mathrm{U}$ & $\mathrm{U}$ & D & $\mathrm{D}$ \\
\hline Sporobolus spicatus & $\mathbf{U}$ & $\mathrm{U}$ & $\mathrm{U}$ & D & $\mathrm{U}$ & I & $\mathrm{U}$ & D \\
\hline Tetrapogon tenellus & $\mathrm{U}$ & 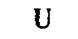 & I & $\mathbf{S}$ & $\mathrm{U}$ & $\mathrm{U}$ & I & D \\
\hline Themeda triandra & $\mathrm{U}$ & $\mathbf{U}$ & D & $\mathrm{D}$ & D & $\mathrm{D}$ & $\mathrm{D}$ & D \\
\hline Tricholaena teneriffae & 1 & I & D & $\mathrm{D}$ & $\mathrm{U}$ & 1 & D & $\mathrm{D}$ \\
\hline \multicolumn{9}{|l|}{ Broadleaf herbaceous plants } \\
\hline Abutilon hirtum & $\mathrm{U}$ & $\mathbf{U}$ & $\mathbf{U}$ & $\mathrm{U}$ & $\mathrm{U}$ & $\mathrm{U}$ & $\mathrm{u}$ & $\mathbf{u}$ \\
\hline Abutilon sp.* & $\mathrm{U}$ & I & $\mathbf{U}$ & I & I & D & I & $\mathrm{D}$ \\
\hline Acalypha indica & $\mathrm{U}$ & U & $\mathrm{U}$ & $\mathrm{u}$ & D & $\mathrm{u}$ & $\mathrm{U}$ & $\mathbf{u}$ \\
\hline Acalypha racemosa & $\mathrm{U}$ & $\mathrm{U}$ & D & $\mathrm{U}$ & $\mathrm{D}$ & $\mathrm{U}$ & $\mathrm{D}$ & $\mathbf{U}$ \\
\hline Aerva persica & $\mathrm{U}$ & $\mathrm{U}$ & $\mathrm{U}$ & $\mathrm{U}$ & $\mathrm{U}$ & $\mathrm{u}$ & $\mathrm{u}$ & $\mathbf{u}$ \\
\hline Barleria argentea & I & I & I & I & I & I & $\mathrm{U}$ & $\mathbf{U}$ \\
\hline Becium oboratum & U & $\mathrm{U}$ & $\mathrm{u}$ & $\mathrm{U}$ & $\mathbf{U}$ & $\mathrm{U}$ & $\mathrm{u}$ & $\mathbf{U}$ \\
\hline Bidens incumbens & $\mathrm{U}$ & $\mathbf{U}$ & $\mathrm{U}$ & $\mathbf{U}$ & I & $\mathrm{U}$ & $\mathrm{u}$ & $\mathrm{u}$ \\
\hline Blepharis linaarifolia & VD & D & $\mathbf{U}$ & $\mathbf{U}$ & VD & $\mathrm{U}$ & I & $\mathbf{U}$ \\
\hline Cleome allamanii & 1 & U & 1 & $\mathrm{U}$ & I & $\mathrm{U}$ & I & $\mathbf{u}$ \\
\hline Cleome brachycarpa & $\mathrm{D}$ & $\mathrm{U}$ & i & $\mathrm{U}$ & $\mathrm{D}$ & $\mathrm{U}$ & $\mathrm{U}$ & $\mathrm{u}$ \\
\hline Cleome tenella & $\mathrm{U}$ & $\mathrm{U}$ & 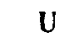 & $\mathrm{U}$ & $\mathrm{U}$ & $\mathrm{U}$ & $\mathrm{u}$ & $\mathrm{U}$ \\
\hline Cleome usambarica & D & $\mathrm{U}$ & $\mathrm{U}$ & $\mathrm{U}$ & D & $\mathrm{U}$ & I & i) \\
\hline Commelina africana & D & D & VD & VD & D & D & VD & VD \\
\hline Cammelina benghalensis & D & D & VD & VD & D & D & VD & VD \\
\hline Conyza phrrhopappa & $\mathrm{U}$ & $\mathbf{U}$ & $\mathbf{U}$ & $\mathrm{U}$ & U & I & $\mathrm{U}$ & I \\
\hline Conyza stricta & $\mathrm{U}$ & $\mathrm{U}$ & $\mathrm{U}$ & $\mathrm{U}$ & $\mathrm{U}$ & I & $\mathrm{u}$ & I \\
\hline Crossandra sp.* & $\mathrm{U}$ & $\mathbf{U}$ & $\mathrm{U}$ & $\mathrm{U}$ & $\mathbf{U}$ & I & $\mathrm{U}$ & $\mathrm{U}$ \\
\hline Crolalaria deserticolor & $\mathrm{U}$ & $\mathrm{U}$ & $\mathrm{U}$ & $\mathrm{U}$ & U & $\mathbf{U}$ & $\mathrm{u}$ & $\mathrm{U}$ \\
\hline Crotalaria massaiensis & U & U & D & 1 & D & I & D & I \\
\hline Cynoglossum coeruleum & $\mathrm{U}$ & $\mathrm{U}$ & $\mathrm{U}$ & $\mathrm{u}$ & $\mathrm{U}$ & U & $\mathrm{u}$ & $\mathrm{U}$ \\
\hline Digera muricata & $\mathrm{U}$ & $\mathrm{U}$ & I & $\mathrm{U}$ & I & $\mathrm{U}$ & $\mathrm{u}$ & $\mathrm{U}$ \\
\hline Euphorbia inaequilatera & $\mathrm{U}$ & $\mathrm{U}$ & D & $\mathrm{U}$ & D & $\mathrm{U}$ & $\mathrm{U}$ & $\mathrm{U}$ \\
\hline Geigeria acaulis & U & $\mathrm{U}$ & $\mathrm{u}$ & $\mathrm{u}$ & u & $\mathrm{U}$ & $\mathrm{U}$ & $\mathrm{u}$ \\
\hline Gesikia pharnacoides & $\mathrm{U}$ & $\mathbf{U}$ & D & $\mathbf{U}$ & D & $\mathrm{U}$ & I & $\mathrm{U}$ \\
\hline Gloriosa superba & U & $\mathrm{U}$ & $\mathrm{U}$ & $\mathrm{u}$ & $\mathrm{U}$ & $\mathrm{U}$ & $\mathrm{U}$ & $\mathrm{U}$ \\
\hline Gynandropsis cleome & U & $\mathbf{U}$ & $U$ & $\mathbf{U}$ & $\mathbf{U}$ & $\mathrm{U}$ & $\mathrm{u}$ & $\mathrm{u}$ \\
\hline Heliotropium sp.* & I & U & $\mathrm{U}$ & $\mathrm{u}$ & 1 & $\mathrm{U}$ & $\mathrm{u}$ & $\mathrm{U}$ \\
\hline Heliltropium steudneri & $\mathrm{U}$ & $\mathrm{U}$ & $\mathrm{U}$ & $\mathrm{U}$ & $\dot{U}$ & U & $\mathrm{u}$ & $\mathrm{U}$ \\
\hline Hermania borannensis & D & $\mathrm{U}$ & $\mathrm{D}$ & $\mathrm{U}$ & D & $\mathrm{U}$ & $\mathrm{D}$ & $\mathrm{U}$ \\
\hline Hermanis kirkii & $\mathrm{U}$ & $\mathrm{U}$ & $\mathrm{U}$ & $\mathrm{U}$ & 1 & $\mathrm{U}$ & $\mathrm{U}$ & $\mathrm{U}$ \\
\hline Indigofera brevicalyx & D & $\mathrm{U}$ & D & $\mathrm{U}$ & $\mathbf{D}$ & $\mathrm{U}$ & D & $\mathrm{U}$ \\
\hline Indigofera coerulea & VD & VD & D & D & VD & VD & $\mathrm{u}$ & I \\
\hline Indigofera hochsteteri & U & U & U & $\mathrm{U}$ & I & $\mathrm{U}$ & $\mathrm{u}$ & $\mathrm{U}$ \\
\hline Ipomoea cordofana & D & $\mathrm{U}$ & I & $\mathrm{U}$ & $\mathrm{U}$ & $\mathrm{U}$ & U & $\mathrm{u}$ \\
\hline Ipomoea erythrocephala & I & I & i & 1 & I & I & I & I \\
\hline Ipomoea kotschyana & $\mathrm{U}$ & $\mathrm{U}$ & U & $\mathrm{U}$ & 1 & $\dot{U}$ & $\mathrm{U}$ & $\mathrm{U}$ \\
\hline Ipomoea sp.* & D & $\mathrm{u}$ & D & $\mathrm{U}$ & $\mathrm{D}$ & $u$ & I & $\mathrm{U}$ \\
\hline Jasminum sp.* & I & $\mathrm{U}$ & I & $\mathrm{U}$ & I & $\mathrm{U}$ & $\mathrm{U}$ & $\mathrm{U}$ \\
\hline Justicia diclipteroides & D & I & I & $\mathrm{U}$ & D & I & $\mathrm{U}$ & $\mathrm{U}$ \\
\hline
\end{tabular}


Justicia erigua

Kouhatia caespitosa

Leucas sp.*

Leucas urticifolia

Limeum prastermissum

Limeum sp.

Mollugo cerviana

Monsonia senegalensis

Ocimum basilicum

Ocimum sp.*

Oldenlandia fastigiata

Oxygonum sinuatum

Phyllanthus fischeri

Phyllanthus rotundifolius

Phyllanthus sp.*

Plectranthus sylvestris

Portulaca oleracea

Portulaca quadrifida

Pupalia lappacea

Senecio lyratripatitus

Sesamum alatum

Sesamum sp.*

Solanum dubium

Stricta sp.*

Tephrosia emeroides

Tribulus terrestris

Vernonia sp.*

Zygophyllum simplex

Annual grasses and sedges

Aristida adscensionis

Aristida mutabilis

Brachiaria deflexa

Brachiaria leersiodes

Brachiaria ovalis

Cenchrus ciliaris

Chloris roxburghiana

Chloris virgata

Cyperus bulbosus

Cyperus laevigatus

Cyperus sp.*

Digitaria sp.*

Digitaria velutera

Drake-Brockmania somalensis

Enneapogon cenchroides

Enneapogon desvauxii

Eragrostis cilianensis

Eragrostis sp.*

Heteropogon contortus

Kyllinga alba

Leptothrium senegalense

Lintonia nutans

Lintonia sp.*

Microchloa kunthii

Oropetium minimum

Pennisetum mezianum

Setaria pallidefusca

Setaria plicatilis

Setaria verticillata

Sorghum sp. (1)*

Sorghum sp.(11)*

Sorghum versicolor

Sporobolus sp. (1)*

Spurobolus sp. (11 small)*

Stipagrostis uniplumis

Tetrapogon cenchriformis

Tetrapogon spatheceus

Tragus berteronianus

Other important species (Creepers, Climbers etc.) Cissus rotundifolia

Euphorbia samburuensis

Euphorbia tirucalli

Vigna membranacea

$\begin{array}{ll}U & \text { I } \\ U & D \\ U & I \\ U & U \\ U & U \\ U & U \\ U & U \\ U & U \\ U & U \\ U & U \\ U & U \\ U & I \\ U & U \\ U & U \\ U & U \\ I & I \\ U & U \\ U & U \\ U & U \\ U & U \\ U & U \\ U & U \\ U & U \\ U & U \\ I & U \\ U & U \\ U & I \\ U & U\end{array}$

D

D

*Specific name not determined. 
The annual plants require special consideration. They contribute to the diets of livestock, especially cattle and sheep, either in their green form in the wet season or in the "standing dead" and litter form in the dry season. Because their presence is unreliable however, they are not normally used for assessing condition. Blepharis linearifolia is highly desirable by camels in its dry form, however.

\section{Trees and Shrubs}

During wet seasons when a flush of green leaves appears, more than $50 \%$ of the trees were found to be utilized by camels and goats (Table 1). There are no records of sheep or cattle consuming trees or large shrubs in the wet season. Since most trees are deciduous, and lose their leaves in the dry season, they are not consumed by livestock. Approximately $50 \%$ of the tree species, however, are known to be utilized by camels, $50 \%$ by goats, and $16 \%$ by sheep, in the dry season. Even cattle have been observed to feed on Acacia tortilis flowers, and Grewia bicolor and Boscia angustifolia which remain green longer than other plant species. Kayongo-Male et al. (1981) recorded Cadaba species as constituting $8 \%$ of the cattle diets in late October 1981 in the Ngurunit/Lependera area. Acacia tortilis pods are known to be eaten by goats and sheep.

Smaller shrubs (about 2 metres high) form an important part of camel and goat diets (Table 1). They are especially browsed in the wet season when about $55 \%$ of the total number of shrub species observed are eaten by camels and $62 \%$ by goats. Sheep and cattle eat approximately $22 \%$ and $6 \%$ of this plant category, respectively, in the wet season. (Table 2).

Dwarf shrubs form a major component of the vegetation in the
IPAL study area where they are frequently dominant (Table 1). Dwarf shrubs are important camel and goat browse in all seasons and are also occasionally browsed by cattle and sheep during periods of food scarcity. The most important species are Acalypha fruticosa, Barleria eranthemoides, Barleria proxima, Indofera spinosa, Justicia cearulea, Justicia pinguor and Plectranthus igniarus. It is common for species of low palatability in the wet season to become desirable in the dry period as illustrated by Crotalaria laburnifolia. and Satureja abyssinica. Duosperma eremophilum. which is one of the most common dominants of the dwarf shrubs, is only occasionally browsed by camels, goats, and cattle. Other important dwarf shrub species, both in terms of availability and desirability, are Heliotropium albohispidum and Sericocomopsia hildebrandtii.

\section{Perennial Grasses}

Perennial grasses in the IPAL study area are mainly found on the mountains, though they also occur in limited areas of the arid plains. They form the most important plant group for sheep and cattle (Table 1). Certain species have been included in this group because they tend to be either annual or perennial. Lewis (1977) reported similar physiology for Eragrostis cilianesis. More than $90 \%$ of the observed perennial grasses were desirable to both sheep and cattle in all seasons. The most desirable plants in this group for sheep and cattle was Dichanthium insculptum (Table 1). In the case of goats, as much as $60 \%$ of the perennial grasses present were found to be desirable (Table 2). Even for camels, which are generally considered to be nongrazers, our observations indicated that about $14 \%$ and $19 \%$ of the total number of perennial grasses were

Table 2. Desirability rating of the various plant species as percentage (\%) of the total species in each class for different livestock species.

\begin{tabular}{|c|c|c|c|c|c|c|c|c|}
\hline & \multicolumn{4}{|c|}{ Wet Season } & \multicolumn{4}{|c|}{ Dry Season } \\
\hline & VD & D & 1 & $\mathbf{u}$ & VD & $\mathrm{D}$ & I & $\mathbf{U}$ \\
\hline \multicolumn{9}{|l|}{ Camels } \\
\hline Tree and large shrubs & 2 & 26 & 29 & 43 & 2 & 24 & 14 & 60 \\
\hline Shrubs & 6 & 33 & 17 & 44 & - & 17 & 22 & 61 \\
\hline Dwarf Shrubs & 6 & 41 & 15 & 38 & 3 & 41 & 29 & 27 \\
\hline Perennial grasses & - & - & 14 & 86 & - & - & 18 & 82 \\
\hline Herbs & 3 & 16 & 10 & 71 & 2 & 4 & 9 & 85 \\
\hline Annual grasses & - & - & 5 & 95 & 5 & - & 24 & 71 \\
\hline Others & 7 & 43 & 29 & 21 & 14 & - & - & 86 \\
\hline \multicolumn{9}{|l|}{ Sheep } \\
\hline Trees and large shrubs & - & - & - & 100 & - & 5 & 11 & 84 \\
\hline Shrubs & - & 5 & 17 & 78 & - & - & 11 & 89 \\
\hline Dwarf shrubs & - & 23 & 15 & 62 & - & 12 & 35 & 53 \\
\hline Perennial grasses & 5 & 86 & 5 & $5-$ & 82 & 9 & 9 & \\
\hline Herbs & 3 & 12 & 20 & 63 & 3 & 2 & 10 & 85 \\
\hline Annual grasses & 10 & 74 & 16 & - & 8 & 74 & 13 & 5 \\
\hline Others & 21 & 29 & 14 & 36 & - & 14 & - & 86 \\
\hline \multicolumn{9}{|l|}{ Goats } \\
\hline Trees and large shrubs & 5 & 34 & 13 & 48 & 2 & 21 & 27 & 50 \\
\hline Shrubs & 6 & 22 & 33 & 39 & - & 11 & 33 & 56 \\
\hline Dwarf shrubs & 7 & 33 & 18 & 42 & 1 & 28 & 42 & 29 \\
\hline Perennial grasses & - & 36 & 23 & 41 & - & 23 & 36 & 41 \\
\hline Herbs & 4 & 24 & 32 & 40 & 2 & 4 & 16 & 78 \\
\hline Annual grasses & - & 11 & 42 & 47 & - & 21 & 32 & 47 \\
\hline Others & 14 & 50 & 14 & 22 & 15 & 22 & - & 63 \\
\hline \multicolumn{9}{|l|}{ Cattle } \\
\hline Trees and large shrubs & - & - & - & 100 & - & - & 3 & 97 \\
\hline Shrubs & - & - & 6 & 94 & - & 5 & 12 & 83 \\
\hline Dwarf shrubs & - & 3 & 9 & 75 & - & 12 & 29 & 59 \\
\hline Perennial grasses & 4 & 86 & 5 & 5 & - & 86 & 5 & 9 \\
\hline Herbs & 3 & 5 & 13 & 79 & 3 & 2 & 10 & 85 \\
\hline Annual grasses & 10 & 75 & 13 & 2 & 9 & 61 & 24 & 6 \\
\hline Others & - & 36 & - & 64 & - & 7 & 14 & 79 \\
\hline
\end{tabular}

$V D=$ Very desirable, $\mathrm{D}=$ Desirable, $\mathrm{I}=$ Intermediate, $\mathrm{U}=$ Undesirable. 
eaten by camels in the wet and dry seasons respectively.

\section{Broadleaf Herbaceous Plants}

The greatest proportion of the 68 broadleaf herbaceous plants encountered in our studies are annuals. Thus when they are eaten in the dry seasons, they are usually dried. The species most preferred arc Cammelina africana and Cameliana benghalensis, which are preferred by cattle, sheep, camels, and goats in all seasons (Table 1). Indigofera caerulea on the other hand, was found to be very desirable for camels and goats and desirable for sheep in all seasons, although it was intermediate for cattle in the dry season and undesirable for cattle in the wet season. Despite its spines, Blepharis linaarifolia is eaten by camels in the wet and dry seasons, as well as cattle, sheep, and goats in the wet season. Because many of the species are desirable only in the wet season, they are not available in the dry periods (Table 1 ).

\section{Annual Grasses and Sedges}

The ground layer of the IPAL study area is dominated by annual grasses, notably Aristida spp. Annual grasses compose the bulk of cattle and sheep diets, a substantial proportion of the diet of goats, and a small but significant contribution to the diet of camels (Table 1). Digitaria sp., D. velutina, and Eragrostis sp. are very desirable for cattle and sheep in all seasons. Another highly preferred plant is Chloris virgata. In fact, only a minor percentage of the plants in this category was recorded as undesirable for cattle and sheep, mainly in the dry season. Undesirables include Oropetium minimum and Tragus berteronianus, which are both very small plants that are not readily available or have sharp, prickly seeds. In the dry season, annual grasses and sedges are eaten standing dead or as litter by livestock. In certain arid conditions such as are commonly found in the IPAL study area, this may be the only food available to the animals. All the sedges encountered, Cyperus laevigatus, $C$. bulbosus, C. sp., Kyllinga alba, were desirable for cattle, sheep, and goats, but not for camels.

\section{Other Important Species}

The last category to be considered includes creeping and climbing plants. Since the majority of these plants are droughtdeciduous, their use by livestock is largely confirmed to the wet seasons (Table 1). However, a few of them are utilized by livestock in the dry season since their stems remain fleshy. The most preferred species in this group is Kandrostis gijef which is highly desirable for camels, sheep, goats, and cattle even in its leafless form. Other preferred species are Rhynchosia minima, Rhynchosia sp., Rhynchosia sublobata, Mormodica sessifolia, and Vigna membranacea. The majority of the plants in this catagory are desirable in the wet season, especially for camels, sheep, and goats.

\section{Summary}

A look at the classification indicates that most of the plants in the study area are used by livestock at different times of the year. There is a clear overlap in the diets of camels and goats and also cattle and sheep. Since this area is used traditionally by all these kinds of livestock, diet overlap should be considered while recommending stocking rates. Manipulation of the range for better primary production should also take into account the needs of the different livestock species on a common use range.

\section{Literature Cited}

Dougall, H.W., and A.V. Bogdan. 1958a. Browse plants of Kenya with special reference to those occurring in South Baringo. E. Afr. Agr. For. J. $23: 236-245$.

Dougall, H.W., and A.V. Bogdan. 1958b. The chemical composition of the grasses of Kenya-1. E. Afr. Agr. For. J. 24:17-23.

Dougall, H.W., V.M. Drysdale, and P.E. Glover. 1964. The chemical composition of Kenya browse and pasture herbage. E. Afr. Wildl. J. 2:86-92.
Edwards, K.A., C.R. Field, and I.M.M. Hogg. 1979. A preliminary analysis of climatological data from the Marsabit District of Northern Kenya. IPAL Tech. Rep. No. B-1. UNEP-MAB Integrated Project in Arid Lands, Nairobi.

Field, A.C. 1978. ODM - IPAL sheep and goats project preliminary report on the impact of sheep and goats on the vegetation in the arid zone of Northern Kenya. IPAL Tech. Rep. No. E-2. UNEP-MAP Integrated Project in Arid Lands, Nairobi.

Field, C.R. 1968. Methods of studying the food habits of some wild ungulates in Uganda. Proc. Nutrition Soc. 27:172-177.

Field, C.R. 1975. Climate and the food habits of ungulates on Galana Ranch. E. Afr. Wildl. J. 13:203-220.

Field, C.R. 1978. The food habits of camels in Northern Kenya. IPAL Tech. Rep. No. E-1b. UNBP-MAB Integrated Project in Arid Lands, Nairobi.

Field, C.R. 1979. Preliminary report on ecology and management of camels, sheep and goats in Northern Kenya. IPAL Tech. Rep. No. E-la. UNEP-MAB Integrated Project in Arid Lands, Nairobi.

Gwynne, M.D. 1979. The nutritive value of Acacia pods in relation to Acacia seed distribution by ungulates. E. Afr. Wildl. J., 7:176-178.

Hepper, P.M.L., J.B. Gillet, and M.G. Gilbert. 1981. Annotated checklist of the plants of Mount Kulal, Kenya. IPAL Tech. Rep. No. D-3. UNEPMAB Integrated Project in Arid Lands, Nairobi.

Herlocker, D. 1979a. Vegetation of southwestern Marsabit District, Kenya. IPAL Tech. Rep. No. D-1. UNEP-MAB Integrated Project in Arid Lands, Nairobi.

Herlocker, D. 1979b. Implementing forestry programmes for local community development, southwestern Marsabit District, Kenya. IPAL Tech. Rep. No. D-2c. UNEP-MAB Integrated Project in Arid Lands, Nairobi.

Herlocker, D., and R.A. Dolan. 1980a. Primary productivity of the herb layer and its relation to rainfall. P. 22-27. In: IPAL Tech. Rep. No. A-3. Proc. of a Scientific seminar, Nairobi 24-27 November 1980. UNEPMAB Integrated Project in Arid Lands, Nairohi.

Kayongo-Male, H., and C.R. Field. 1981. Feed quality and utilization by cattle grazing natural pasture in the range of Northern Kenya. (Unpublished).

Lamprey, H.F., D.J. Herlocker, and C.R. Field. 1980. The state of knowledge on browse in East Africa. In: Proc. Intern. Conference on Browse Production: Addis Ababa.

Lewis, J.G. 1977. Report of a short-term consultancy on the grazing ecosystem in the Mt. Kulal region, Northern Kenya. IPAL Tech. Rep. No, E-3. UNEP-MAB Integrated Project in Arid lands, Nairobi.

Lusigi, W.J. 1981. Combatting desertification and rehabilitating degraded production systems in Northern Kenya. IPAL Tech. Rep. A-4.

Said, A.N. 1980. Goat nutrition. P. 74-77. In: IPAL Tech. Rep. No. A-3. Proc. of a scientific seminar, Nairobi, 24-27 November 1980. UNEPMA3 Integrated Project in Arid Lands. Nairobi.

Sands, E.B., D.B. Thomas, J. Knight, and D.J. Pratt. 1970. Preliminary selection of pasture plants for the semi-arid areas of Kenya. E. Afr. Agr. For. J. 36:49-52.

Stewart, D.R.M. 1966. A technique for studying the food preferences of grazing herbivores. Ph. D. Thesis, Univ. East Africa.

Synott, T.J. 1979a. A report on the status, importance and protection of the montane forests. IPAL Tech. Rep. No. D-2a. UNEP-MAB Integrated Project in Arid Lands, Nairobi.

Synott, T.J. 1979b. A report on prospects, problems and proposals for tree planting. IPAL Tech. Rep. No. D-2B. UNEP-MAP Integrated Project in Arid Lands, Nairobi.

Taerum, R. 1970. A note on chemical content of some East African grasses. E. Afr. Agr. For. J. 36:171-176.

Talbot, L.M. 1962. Food peferences of some East African Wild ungulates. E. Afr. Agr. For. J. 27:131-138.

Walther, D., and D.J. Herlocker. 1980 . A preliminary study of the relationship between vegetation, soils and land use within southwestern Marsabit District. P. 41-54. In: IPAL Tech. Rep. No. A-3. Proc. of a scientific seminar, Nairobi, 24-27 November 1980. UNEP-MAB Integrated Project in Arid Lands, Nairobi. 